\title{
Baja incidencia y alta mortalidad por COVID-19 en Cirugía General durante la primera ola de la pandemia
}

\author{
Alberto G. Barranquero ${ }^{1}$, Diego Ramos ${ }^{1}$, Juan Ocaña ${ }^{1}$, Ana Puerta ${ }^{1}$, \\ Alberto Vilar Tabanera ${ }^{1}$, Mariam Bajawi ${ }^{1}$, Víctor Vaello ${ }^{1}$, Luz Divina Juez ${ }^{1}$, \\ Jordi Núñez ${ }^{1}$, Alba G. Chiloeches ${ }^{1}$, Paula Pastor ${ }^{1}$, Ángela Santana Valenciano, \\ Elena Payno ${ }^{1}$, Paula Muñoz Muñoz ${ }^{1}$ y José María Fernández Cebrián ${ }^{1}$
}

'Departamento de Cirugía General y del Aparato Digestivo. Hospital Universitario Ramón y Cajal. Madrid, España.

Recepción 2021-01-20, aceptado 2021-03-15

Correspondencia a: Dr. Alberto G. Barranquero albertober.21@gmail.com

\section{Low incidence and high COVID-19 mortality in a General Surgery during the first wave of the pandemia}

The impact of coronavirus disease (COVID-19) in intervened patients seems to cause large postoperative mortality, although its incidence varies among centres. Primary aim was to evaluate the incidence of COVID-19 on the patients intervened in our General and Digestive Surgery Department, during the maximum impact of the pandemia in Spain. Secondary outcomes were evaluating perioperative mortality, and determining the risk factors for COVID-19 infection. Materials and Method: Retrospective single centre study of consecutive patients undergoing general and gastrointestinal surgical procedures with more than 24 hours of in-hospital stay, from February 1, 2020 to April 30, 2020 in a tertiary referral centre in Madrid, Spain. Results: A total of 441 patients were analysed: 423 were non-COVID-19 patients while 18 of them had COVID-19. Preoperative and operative characteristics were similar for both groups, unless for the American Society of Anesthesiologists grade. The incidence of COVID-19 in our intervened patients was $4.1 \%$. Postoperative mortality was high among surgical patients with COVID-19, with a mortality rate of $22.2 \%$ compared to a $2.8 \%$ in non COVID-19 patients. The risk factors for COVID-19 infection were a prolonged postoperative stay (OR: 1.035 [95\% CI: 1.007-1.065]) and the need of a reintervention (OR: 5.025 [95\% CI: 1.650-15.311]). Conclusion: Surgical interventions during the COVID-19 pandemia resulted in a low infection rate but a high postoperative COVID-19 mortality. The decision to intervene must be carefully balanced against the additional risk for patients in a high transmission setting.

Key words: incidence; mortality, coronavirus, COVID-19, surgery, general surgery.

\section{Resumen}

Introducción y objetivo: La infección por coronavirus (COVID-19) en pacientes intervenidos es causa de importante mortalidad posoperatoria, aunque su incidencia es variable. El objetivo primario fue evaluar la incidencia de COVID-19 en los pacientes intervenidos en nuestro Servicio de Cirugía General y Digestiva, durante el máximo impacto de la pandemia en España. El objetivo secundario fue evaluar la mortalidad perioperatoria y determinar los factores de riesgo para la infección por COVID-19. Materiales y Método: Estudio observacional retrospectivo de pacientes consecutivos sometidos a Cirugía General y Digestiva con ingreso superior a 24 h, del 1 de febrero de 2020 al 30 de abril de 2020 en un hospital terciario de Madrid, España. Resultados: Se analizaron 441 pacientes: 423 sin COVID-19 y 18 con COVID-19. Las características preoperatorias y operatorias fueron similares para ambos grupos, salvo por el grado ASA (American Society of Anesthesiologists). La incidencia de COVID-19 en los pacientes intervenidos fue del 4,1\%. La mortalidad posoperatoria fue elevada, del 22,2\% en pacientes con COVID-19, frente a un 2,8\% en pacientes no COVID-19 (p: 0,003). Los factores de riesgo para la infección por COVID-19 en los pacientes intervenidos fueron una estancia hospitalaria prolongada (OR: 1,035 [95\% CI: 1,007-1,065]) y la reintervención quirúrgica (OR: 5,025 [95\% CI: 1,650-15,311]). Conclusión: Las intervenciones durante la pandemia de COVID-19 causaron una baja tasa de infección con elevada mortalidad posoperatoria. La intervención quirúrgica debe valorarse frente al riesgo adicional para el paciente en contextos de alta transmisión.

Palabras clave: incidencia; mortalidad; coronavirus; COVID-19; cirugía; cirugía general. 


\section{Introducción}

Desde los primeros casos reportados de síndrome respiratorio agudo severo por coronavirus-2 (SARSCoV-2), y tras la declaración como pandemia de la infección por coronavirus (COVID-19) por la Organización Mundial de la Salud ${ }^{1}$, millones de personas han sufrido una infección confirmada por COVID-192. A la fecha, tras América, Europa es la segunda región más afectada a nivel mundial ${ }^{2}$, y España ha sido uno de los países epicentro del brote europeo. Es el décimo país en número absoluto de muertes y el noveno país con mayor número de casos confirmados ${ }^{3}$. La infección por COVID-19 es todavía un problema de primer orden en Estados Unidos, Brasil o México. Algunos países, como España, incluso se acercan a una tercera ola ${ }^{4}$.

La infección por SARS-CoV-2 es grave en un $18,1 \%$ de los casos, con una incidencia de síndrome de distrés respiratorio del adulto (SDRA) del 14,8\% y una mortalidad del $4,3 \%{ }^{5}$. Pacientes con comorbilidad presentan tasas de mortalidad mayores. Hipertensión arterial, diabetes, enfermedad pulmonar obstructiva crónica (EPOC) o cáncer son factores de riesgo para un peor desenlace.

La cirugía se ha visto afectada por la pandemia de COVID-19. Los estudios iniciales indicaron una elevada mortalidad, del $20,5 \%{ }^{7}$ al $66,6 \%{ }^{8}$. Ello promovió que múltiples sociedades quirúrgicas, entre ellas la Asociación Española de Cirugía (AEC), recomendaran una reducción de la actividad quirúrgica en procedimientos electivos o demorables ${ }^{9,10}$, por lo que se estima que más de 28 millones de procedimientos electivos fueron suspendidos en los primeros meses de la pandemia ${ }^{11}$. Las intervenciones quirúrgicas en pacientes con COVID-19 han asociado una tasa de complicaciones pulmonares del $51,2 \%$ y una mortalidad posquirúrgica del $23,8 \%{ }^{12}$.

\section{Objetivo}

El objetivo primario fue evaluar la incidencia de COVID-19 en los pacientes intervenidos en nuestro Servicio de Cirugía General y Digestiva, durante el máximo impacto de la pandemia en España. El objetivo secundario fue evaluar la mortalidad perioperatoria del periodo a estudio y determinar los factores de riesgo para la infección por COVID-19 en los pacientes intervenidos.

\section{Materiales y Método}

Este artículo sigue las recomendaciones STROBE (Strengthening the Reporting of Observational
Studies in Epidemiology) para estudios observacionales ${ }^{13}$.

\section{Diseño del estudio}

Estudio observacional retrospectivo unicéntrico.

\section{Participantes}

Se incluyeron todos los pacientes consecutivos mayores de 18 años de edad sometidos a Cirugía General y Digestiva con ingreso superior a $24 \mathrm{~h}$ desde el 1 de febrero de 2020 al 30 de abril de 2020 en un centro hospitalario de tercer nivel en Madrid, España.

Fueron excluidos los pacientes con una cirugía inicial previa al reclutamiento y reintervención durante el periodo de estudio y los pacientes perdidos en el seguimiento.

Nuestra institución atiende a una población en torno a 600.000 personas, con 900 camas de hospitalización y 58 camas de Cuidados Intensivos. Los primeros casos de COVID-19 fueron detectados a inicios de marzo, con siete pacientes hospitalizados. El 13 de marzo de 2020, el Gobierno de España declaró el confinamiento de la población debido a una elevada transmisión comunitaria de la infección COVID-19. El 18 de marzo, un tercio de los recursos del hospital ya estaban dedicados en exclusiva a pacientes con COVID-19. El 30 de marzo se alcanzó el pico, con 1.000 pacientes hospitalizados y 103 pacientes en UCI. En este periodo, los quirófanos se habilitaron como áreas de Cuidados Intensivos y, desde mediados de marzo, los servicios médicos con personal de otras especialidades como refuerzo. $\mathrm{La}$ situación comenzó a mejorar hacia la tercera semana de abril, y para el final del mes de abril quedaban 207 pacientes hospitalizados y 45 en UCI, mientras la actividad quirúrgica volvió a aumentar.

Este estudio fue aprobado por el Comité de Ética de Investigación (número de registro: 134-20) y realizado de acuerdo con los estándares éticos de la Declaración de Helsinki de 1975.

\section{Variables}

Se extrajeron los datos mediante la revisión de la historia clínica electrónica. La infección COVID-19 se consideró confirmada con una prueba positiva de reacción en cadena de la polimerasa con transcriptasa inversa (RT-PCR) del exudado nasofaríngeo. La infección fue considerada probable basada en hallazgos clínicos y radiológicos.

Las variables preoperatorias fueron: sexo, edad, índice de masa corporal, hábito tabáquico, comorbilidades previas (hipertensión arterial, diabetes, EPOC, apnea obstructiva del sueño, insuficiencia 
cardiaca, cardiopatía isquémica, ictus, enfermedad renal crónica, neoplasia activa), tratamiento oncológico o tratamiento inmunosupresor concomitante, categoría ASA (American Society of Anesthesiologists) y pruebas de imagen de tórax preoperatorias (radiografía y tomografía computarizada).

Los datos operatorios fueron: región intervenida, anestesia, dispositivo para el manejo de la vía aérea utilizado, momento de la cirugía (emergente, urgente o electiva), cirugía oncológica, abordaje y duración del procedimiento. Los procedimientos realizados por subespecialidad quirúrgica incluyen en cirugía colorrectal: apendicectomía, hemicolectomía derecha o izquierda, colectomía total, resección anterior baja de recto, amputación abdominoperi-

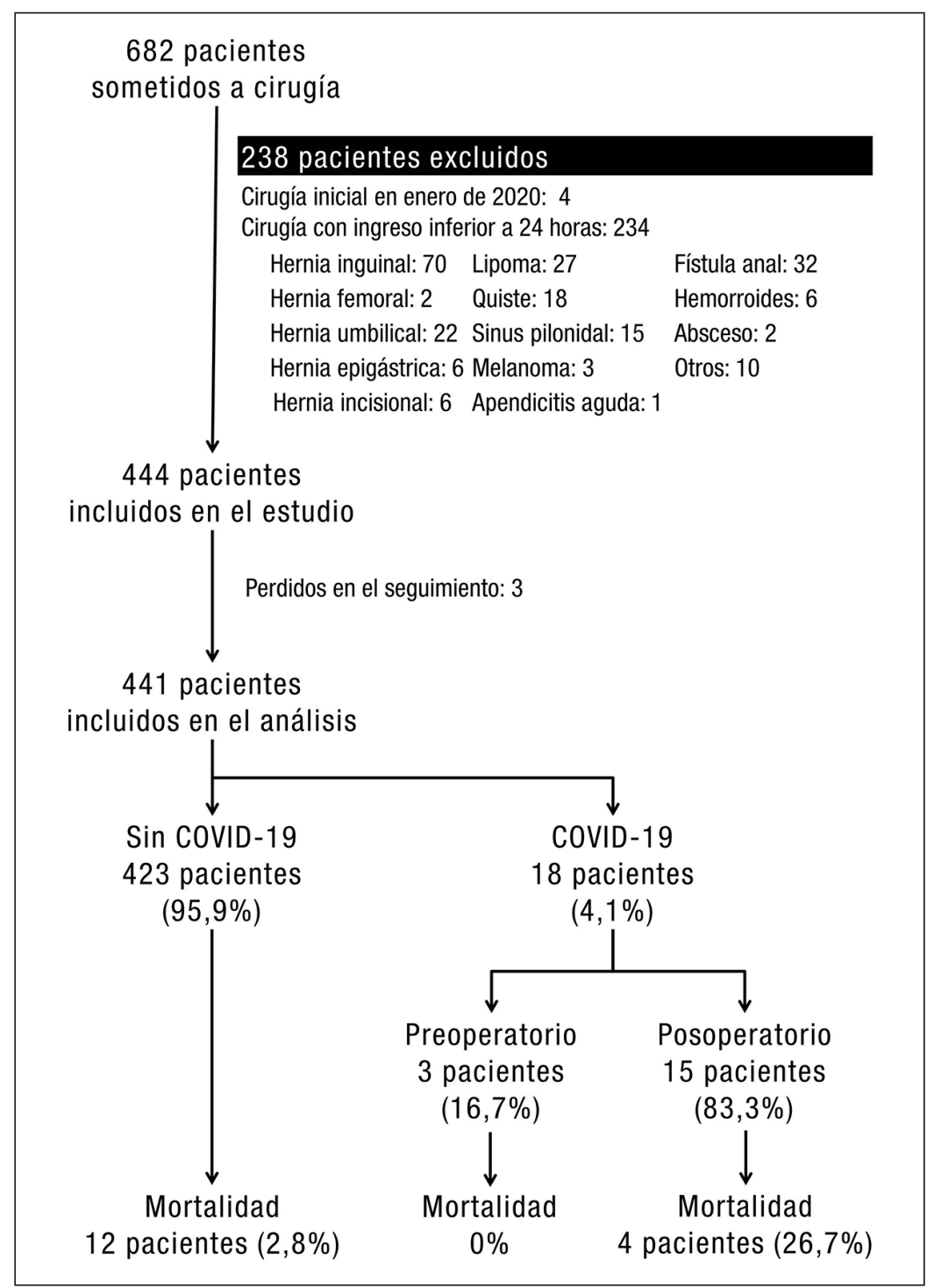

Figura 1. Diagrama de flujo de los pacientes a estudio. neal, creación de colostomía, reconstrucción del tránsito y cirugía perianal; en cirugía hepatobiliopancreática: colecistectomía, resección hepática, trasplante hepático, duodenopancreatectomía cefálica, pancreatectomía corporocaudal y esplenectomía; en cirugía gastroesofágica: funduplicatura, bypass gástrico, gastrectomía vertical, gastrectomía total o subtotal y sutura de úlcera péptica; en cirugía de mama: tumorectomía, mastectomía, biopsia selectiva de ganglio centinela y linfadenectomía axilar; en cirugía endocrina: hemitiroidectomía, tiroidectomía total, paratiroidectomía y linfadenectomía cervical; en cirugía de pared abdominal: hernioplastía, y cirugía de la hernia incisional. Se incluyeron también otros procedimientos realizados en el servicio.

Las variables posoperatorias fueron: estancia hospitalaria, morbimortalidad global, complicaciones específicas (incluyendo complicaciones infecciosas y hemorrágicas), complicaciones de acuerdo con la clasificación de Clavien-Dindo ${ }^{14}$, necesidad de reintervención, soporte ventilatorio posoperatorio, necesidad de ingreso en UCI y reingresos en los siguientes 30 días.

\section{Análisis estadístico}

Las variables categóricas fueron descritas como valor absoluto y porcentaje. Las variables cuantitativas fueron descritas con la media y la distribución estándar ( $\mathrm{sd}$ ) si seguían una distribución normal, y con la mediana y el rango intercuartílico (IQR) si seguían una distribución no normal. La distribución de las variables se determinó mediante test de ShapiroWilk. Para el contraste de hipótesis, se empleó el $\chi 2$ en las variables categóricas, la $\mathrm{t}$ de Student en las variables cuantitativas con distribución normal y la U de Mann-Whitney en las variables cuantitativas con distribución no normal. Para evaluar los factores de riesgo para COVID-19 se empleó un modelo de regresión logística multivariante. La significación estadística se situó en $<0,05$. Se utilizó el SPSS Statistics $23^{\circledR}$ (IBM, Chicago, IL).

\section{Resultados}

\section{Participantes}

Desde el 1 de febrero de 2020 al 30 de abril de 2020, 682 pacientes fueron sometidos a Cirugía General y Digestiva en nuestra institución. Tras excluir 238 pacientes, fundamentalmente por cirugía con ingreso inferior a 24 h, y 3 pérdidas en el seguimiento, 441 pacientes fueron incluidos en el análisis. En el periodo de estudio, 18 pacientes fueron diagnosticados de COVID-19 (Figura 1). 


\section{Datos preoperatorios}

Las características basales en el momento de la cirugía se muestran en la Tabla 1 . No hubo diferencias por edad ni sexo, ni en relación al hábito tabáquico, los factores de riesgo cardiovascular habituales, enfermedades respiratorias ni otras comorbilidades. Se encontraron diferencias significativas en el grado ASA, con una mayor proporción de pacientes ASA 3 en el grupo COVID-19 (10 pacientes $[55,6 \%]$ ), y una mayor proporción de pacientes ASA 2 en el grupo sin COVID-19 (212 pacientes $[50,1 \%]$ ).

La evaluación de tórax preoperatoria mediante radiografía no mostró diferencias entre grupos, mientras que a una mayor proporción de pacientes en el grupo COVID-19 se les realizó un TC de tórax preoperatorio, con mayor número de hallazgos patológicos $(22,2 \%$ vs. 4,5\%).

\section{Datos quirúrgicos}

Los datos quirúrgicos se encuentran en la Tabla 2. No se encontraron diferencias analizando la región intervenida. La mayor parte de los pacientes COVID-19 fueron sometidos a una cirugía colorrectal (11 of 18 [61,1\%]) mientras que ninguno de los pacientes sometidos a una cirugía de mama o endocrina fueron diagnosticados de COVID-19. Hubo mayor proporción de cirugías emergentes $(22,2 \%$ vs. $3,9 \%)$ y urgentes $(16,7 \%$ vs. $3,9 \%)$ en el grupo COVID-19, aunque estas diferencias no fueron estadísticamente significativas. La proporción de intervenciones abiertas fue también algo superior en el grupo COVID-19 (72,2\% vs. 57,7\%; p: 0,221). Finalmente, no hubo diferencias en relación al tiempo quirúrgico, con una mediana de $80 \mathrm{~min}$ (IQR: 45-142,5).

\section{Incidencia de COVID-19}

Durante el periodo de estudio, 18 de 441 pacientes intervenidos fueron diagnosticados de COVID-19, lo que ofrece una incidencia del 4,1\% (Figura 2). La mayor parte de los pacientes presentaron una infección confirmada por PCR del exudado nasofaríngeo (12 de 18 [66,7\%]), mientras que en el resto el diagnóstico fue basado en los hallazgos radiológicos y clínicos (6 de 18 [33,3\%]). La mediana de seguimiento de la muestra fue de 38 días (IQR: 20-64). Tomando en consideración solo el peor periodo de la pandemia, desde el 1 de marzo de 2020 al 30 de abril de 2020, la incidencia fue del $6,8 \%$ (13 de 179)

La actividad quirúrgica se redujo drásticamente, con una media de 62,8 intervenciones por semana (sd: 4,3) en febrero a 30 (sd: 24,1) y 14 (sd: 2,55)
Tabla 1. Características basales

\begin{tabular}{|c|c|c|c|}
\hline & $\begin{array}{l}\text { No COVID-19 } \\
423 \text { pacientes } \\
\text { n (\%) } \\
\text { Mediana (IQR) }\end{array}$ & $\begin{array}{c}\text { COVID-19+ } \\
18 \text { pacientes } \\
\text { n (\%) } \\
\text { Mediana (IQR) }\end{array}$ & $p$ valor \\
\hline $\begin{array}{l}\text { Sexo } \\
\text { Hombre: Mujer }\end{array}$ & $\begin{array}{c}197: 226 \\
(46,6: 53,4)\end{array}$ & $\begin{array}{c}11: 7 \\
(61,1: 38,9)\end{array}$ & 0,239 \\
\hline $\begin{array}{l}\text { Edad } \\
\text { (años) }\end{array}$ & $\begin{array}{c}61,7 \\
(46,6-73,6)\end{array}$ & $\begin{array}{c}65,4 \\
(55,9-78,7)\end{array}$ & 0,143 \\
\hline $\begin{array}{l}\text { IMC } \\
\left(\mathrm{kg} / \mathrm{m}^{2}\right)\end{array}$ & $\begin{array}{c}27 \\
(23,7-30,69)\end{array}$ & $\begin{array}{c}28,4(25,4- \\
32,13)\end{array}$ & 0,185 \\
\hline $\begin{array}{l}\text { Tabaquismo } \\
\text { No fumador } \\
\text { Ex-fumador } \\
\text { Fumador activo }\end{array}$ & $\begin{array}{c}294(69,5) \\
76(18) \\
53(12,5)\end{array}$ & $\begin{array}{l}13(72,2) \\
2(11,1) \\
3(16,7)\end{array}$ & 0,701 \\
\hline Hipertensión & $159(37,6)$ & $7(38,9)$ & 0,911 \\
\hline Diabetes mellitus & $61(14,4)$ & $1(5,6)$ & 0,490 \\
\hline Asma & $27(6,4)$ & 0 & 0,616 \\
\hline EPOC & $19(4,5)$ & 0 & 1,000 \\
\hline Apnea obstructiva del sueño & $27(6,4)$ & 0 & 0,616 \\
\hline Insuficiencia cardiaca & $13(3,1)$ & 0 & 1,000 \\
\hline Cardiopatía isquémica & $27(6,4)$ & 0 & 0,335 \\
\hline Ictus & $12(2,8)$ & $1(5,6)$ & 0,423 \\
\hline Enfermedad renal crónica & $10(2,4)$ & $2(11,1)$ & 0,082 \\
\hline Neoplasia activa & $130(30,7)$ & $5(27,8)$ & 0,790 \\
\hline Tratamiento neoadyuvante & $31(7,3)$ & $1(5,6)$ & 1,000 \\
\hline Tratamiento inmunosupresor & $17(4)$ & 0 & 1,000 \\
\hline $\begin{array}{l}\text { Categoría ASA } \\
\text { Grado } 1 \\
\text { Grado } 2 \\
\text { Grado } 3 \\
\text { Grado } 4\end{array}$ & $\begin{array}{c}78(18,4) \\
212(50,1) \\
122(28,8) \\
11(2,6)\end{array}$ & $\begin{array}{c}3(16,7) \\
3(16,7) \\
10(55,6) \\
2(11,6)\end{array}$ & 0,006 \\
\hline $\begin{array}{l}\text { Radiografía de tórax preoperatoria } \\
\text { No realizada } \\
\text { Normal } \\
\text { Consolidación } \\
\text { Otros hallazgos }\end{array}$ & $\begin{array}{c}108(25,5) \\
295(69,7) \\
3(0,7) \\
17(4)\end{array}$ & $\begin{array}{c}3(16,7) \\
13(72,2) \\
1(5,6) \\
1(5,6)\end{array}$ & 0,163 \\
\hline $\begin{array}{l}\text { TC de tórax preoperatorio } \\
\text { No realizado } \\
\text { Normal } \\
\text { Consolidación } \\
\text { Opacidad en vidrio deslustrado } \\
\text { Otros hallazgos }\end{array}$ & $\begin{array}{c}309(73,4) \\
93(22,1) \\
1(0,2) \\
4(1) \\
14(3,3)\end{array}$ & $\begin{array}{l}7(38,9) \\
7(38,9) \\
1(5,6) \\
1(5,6) \\
2(11,1)\end{array}$ & 0,000 \\
\hline
\end{tabular}


Tabla 2. Datos quirúrgicos

\begin{tabular}{|c|c|c|c|}
\hline & $\begin{array}{c}\text { No COVID-19 } \\
423 \text { pacientes } \\
\text { n }(\%) \\
\text { Mediana (IQR) }\end{array}$ & $\begin{array}{c}\text { COVID-19+ } \\
18 \text { pacientes } \\
\text { n }(\%) \\
\text { Mediana (IQR) }\end{array}$ & p valor \\
\hline \multicolumn{4}{|l|}{ Región intervenida } \\
\hline Cabeza & $4 \quad(0,9)$ & & \\
\hline Cuello & $50(11,8)$ & $1(5,6)$ & \\
\hline Mama & $11(2,6)$ & & \\
\hline Pared abdominal & $40 \quad(9,5)$ & $2(11,1)$ & \\
\hline Cavidad abdominal & $270(63,8)$ & $13(72,2)$ & 0,973 \\
\hline Pelvis & $42(9,9)$ & $2(11,1)$ & \\
\hline Miembros superiores & $2(0,5)$ & 0 & \\
\hline Miembros inferiores & $4 \quad(0,9)$ & 0 & \\
\hline \multicolumn{4}{|l|}{ Subespecialidad quirúrgica } \\
\hline Colorrectal & $162(38,3)$ & $11(61,1)$ & \\
\hline Hepatobiliopancreática & $96(22,7)$ & $1(5,6)$ & \\
\hline Gastroesofágica & $42(9,9)$ & $2(11,1)$ & 0,124 \\
\hline Mama y cirugía endocrina & $63(14,9)$ & & \\
\hline Pared abdominal & $43(10,2)$ & $3(16,7)$ & \\
\hline Otras cirugías & 17 (4) & $1(5,6)$ & \\
\hline Anestesia & & & 0,216 \\
\hline Local & $7 \quad(1,7)$ & $1 \quad(5,6)$ & \\
\hline Regional & $37(8,1)$ & & \\
\hline General & $379(89,6)$ & $17(94,4)$ & \\
\hline Intubación & & & 1,000 \\
\hline Mascarilla laríngea & $15 / 379(4,3)$ & 0 & \\
\hline Intubación orotraqueal & $364 / 379(95,7)$ & $17 / 17(100)$ & \\
\hline Tipo de cirugía & & & 0,527 \\
\hline Emergente $(<24$ h) & $15(3,9)$ & $4(22,2)$ & \\
\hline Urgente $(<72 \mathrm{~h})$ & $15 \quad(3,9)$ & $3(16,7)$ & \\
\hline Electiva & $365(96,1)$ & $11(61,1)$ & \\
\hline Cirugía oncológica & $115(27,2)$ & $3(16,7)$ & 0,442 \\
\hline Abordaje & & & 0,221 \\
\hline Abierto & $224(57,7)$ & $13(72,2)$ & \\
\hline Laparoscópico & $179(42,3)$ & $5(27,8)$ & \\
\hline Tiempo quirúrgico (min) & $80(45-140)$ & $77,5(48,8-202,5)$ & 0,252 \\
\hline
\end{tabular}

intervenciones por semana durante los meses de marzo y abril, respectivamente.

\section{Resultados posoperatorios}

El periodo posoperatorio se resume en la Tabla 3. La mortalidad posoperatoria fue notablemente superior en los pacientes con COVID-19, con una mortalidad del 22,2\% (4 de 18), comparada con un 2,8\% (12 de 423) en los pacientes sin COVID-19 (p: 0,003). Los pacientes con infección por COVID-19 presentaron 9,785 veces (IC 95\%: 2,802-34,179) mayor riesgo de muerte que los pacientes sin infección COVID-19.

Los pacientes COVID-19 presentaron una mayor estancia hospitalaria (8,5 días [IQR: 4,518] vs. 3 días [IQR: 1-8]), mayor reingreso $(29,4 \%$ vs. $4,8 \%)$ y mayor proporción de reintervenciones $(27,8 \%$ vs. $6,9 \%)$. Los pacientes con $\mathrm{CO}$ VID-19 requirieron un mayor soporte respiratorio posquirúrgico, especialmente en forma de mascarilla facial o reservorio $(22,2 \%$ vs. $11,4 \%)$ y mayor proporción de ingreso en UCI posoperatorio, aunque planeado desde el quirófano

Entre los pacientes con COVID-19 hubo una mayor proporción de neumonía $(77,8 \%$ vs. $2,6 \%)$. De ellos, cuatro presentaron una neumonía grave $(22,2 \%)$ y dos tuvieron un síndrome de distrés respiratorio del adulto $(11,1 \%)$. La mayor proporción de complicaciones posoperatorias en el grupo COVID-19 se vio reflejada también en la clasificación de Clavien-Dindo, donde un 44,4\% tuvieron complicaciones mayores, comparado con un $12,5 \%$ de grupo sin COVID-19.

Los factores de riesgo para el desarrollo de infec-
Figura 2. Evolución temporal del número de cirugías por semana (línea negra) frente al número de casos de COVID-19 (línea gris).

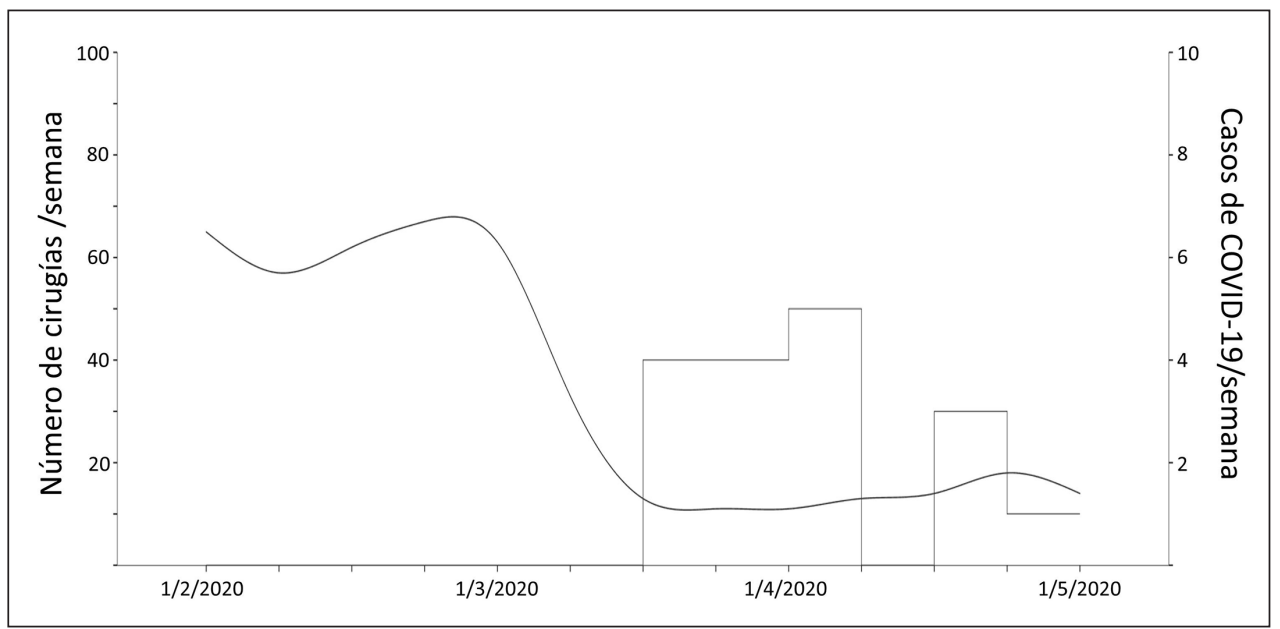


ción por COVID-19 en los pacientes intervenidos en nuestro Servicio de Cirugía General en el análisis multivariante (Tabla 4) fueron una estancia posoperatoria prolongada (OR: 1,035 [IC 95\%: 1,007$1,065])$ y la necesidad de reintervención quirúrgica (OR: 5,025 [IC 95\%: 1,650-15,311]).

\section{Discusión}

\section{Resultados principales}

Las intervenciones quirúrgicas durante la pandemia resultaron en una incidencia de COVID-19 del $4,1 \%$ en nuestra institución. La mortalidad posoperatoria fue elevada, del 22,2\% (4 de 18) en los pacientes con COVID-19, frente a un 2,8\% (12 de 423) en los pacientes sin COVID-19 (p: 0,003). Los factores de riesgo para infección por COVID-19 en los pacientes intervenidos fueron una estancia posoperatoria prolongada (OR: 1,035 [IC 95\%: 1,007$1,065])$ y la necesidad de una reintervención quirúrgica (OR: 5,025 [IC 95\%: 1,650-15,311]).

\section{Fortalezas y limitaciones}

Uno de los puntos fuertes de este estudio es la comparación con un grupo de pacientes no COVID. Esto reduce la limitación de la comparación entre pacientes COVID-19 con manifestaciones leves y graves de la enfermedad ${ }^{7,12}$, o la comparación con una muestra de controles históricos ${ }^{19}$. Además, ofrece mayor evidencia que las series de casos publicadas $^{7,8,20}$.

Entre las limitaciones del estudio encontramos que el diseño impide determinar causalidad, lo que es patente al analizar la estancia hospitalaria prolongada como factor de riesgo para infección por COVID-19. Algunos pacientes presentaron infección por COVID-19 debido a una reintervención con ingreso prolongado posterior, mientras que otros tuvieron una infección por COVID-19 que prolongó su estancia hospitalaria.

\section{Interpretación}

La actividad quirúrgica no demorable ha tenido que ser mantenida durante la pandemia de COVID-19 incluso en países con elevada transmisión comunitaria. Algunos estudios han indicado que la actividad en cirugía digestiva ${ }^{15}$, mínimamente invasiva $^{16}$ o cirugía endocrina ${ }^{17}$ ha podido ser realizada con una tasa de infección del $0 \%$ en pacientes seleccionados con negatividad de las pruebas preoperatorias, pero esta ha sido la excepción, más que la norma. La mayor incidencia de COVID-19 en una población quirúrgica fue la indicada por
Tabla 3. Evolución posoperatoria

\begin{tabular}{|c|c|c|c|}
\hline & $\begin{array}{l}\text { No COVID-19 } \\
423 \text { pacientes } \\
\text { n }(\%) \\
\text { Mediana } \\
\text { (IQR) }\end{array}$ & $\begin{array}{l}\text { COVID-19+ } \\
18 \text { pacientes } \\
\text { n }(\%) \\
\text { Mediana } \\
\text { (IQR) }\end{array}$ & $\underset{\text { valor }}{\mathbf{p}}$ \\
\hline Estancia posoperatoria (días) & $3(1-8)$ & $8,5(4,5-18)$ & 0,005 \\
\hline Mortalidad & $12(2,8)$ & $4(22,2)$ & 0,003 \\
\hline Morbilidad & $93(22)$ & $18(100)$ & $\mathbf{0 , 0 0 0}$ \\
\hline Reingreso & $20 \quad(4,8)$ & $5(29,4)$ & 0,002 \\
\hline $\begin{array}{l}\text { Complicaciones según Clavien-Dindo } \\
\text { I } \\
\text { II } \\
\text { III } \\
\text { IV } \\
\text { V }\end{array}$ & $\begin{array}{ll}21 & (5) \\
19 & (4,5) \\
25 & (5,9) \\
16 & (3,8) \\
12 & (2,8)\end{array}$ & $\begin{array}{l}3(16,7) \\
2(11,1) \\
3(16,7) \\
1 \quad(5,6) \\
4(22,2)\end{array}$ & 0,000 \\
\hline Reintervención & $29(6,9)$ & $5(27,8)$ & 0,008 \\
\hline $\begin{array}{l}\text { Soporte respiratorio posoperatorio } \\
\text { Ninguno } \\
\text { Gafas nasales } \\
\text { Ventimask/reservorio } \\
\text { Ventilación no invasiva } \\
\text { Ventilación invasiva }\end{array}$ & $\begin{array}{r}246(58,3) \\
109(25,8) \\
48(11,4) \\
0 \\
19(4,5)\end{array}$ & $\begin{array}{l}7(38,9) \\
5(27,8) \\
4(22,2) \\
1 \quad(5,6) \\
1 \quad(5,6)\end{array}$ & $\mathbf{0 , 0 0 0}$ \\
\hline $\begin{array}{l}\text { Estancia en UCI posoperatoria } \\
\text { Ninguna } \\
\text { Planeada } \\
\text { No planeada desde el quirófano } \\
\text { No planeada desde la planta }\end{array}$ & $\begin{aligned} 347 & (82) \\
68 & (16,1) \\
3 & (0,7) \\
4 & (0,9)\end{aligned}$ & $\begin{array}{l}1(61,1) \\
7(38,9) \\
0 \\
0\end{array}$ & 0,000 \\
\hline $\begin{array}{l}\text { Complicaciones infecciosas } \\
\text { Infección de herida superficial } \\
\text { Infección profunda del sitio quirúrgico } \\
\text { Perforación gastrointestinal } \\
\text { Fuga anastomótica } \\
\text { Bacteriemia } \\
\text { Neumonía } \\
\text { Shock séptico } \\
\text { Fiebre no especificada }\end{array}$ & $\begin{aligned} 18 & (4,3) \\
12 & (2,8) \\
7 & (1,7) \\
7 & (1,7) \\
2 & (0,5) \\
11 & (2,6) \\
15 & (3,5) \\
3 & (0,7)\end{aligned}$ & $\begin{array}{rr}5 & (27,8) \\
1 & (5,6) \\
1 & (5,6) \\
1 & (5,6) \\
3 & (16,7) \\
14 & (77,8) \\
4 & (22,2) \\
0 & \end{array}$ & $\begin{array}{l}\mathbf{0 , 0 0 1} \\
0,423 \\
0,285 \\
0,285 \\
\mathbf{0 , 0 0 1} \\
\mathbf{0 , 0 0 0} \\
\mathbf{0 , 0 0 5} \\
1,000\end{array}$ \\
\hline $\begin{array}{l}\text { Complicaciones hemorrágicas } \\
\text { Hematoma } \\
\text { Hemorragia } \\
\text { Transfusión sanguínea }\end{array}$ & $\begin{array}{rr}4 & (0,9) \\
7 & (1,7) \\
11 & (2,6)\end{array}$ & $\begin{array}{l}1 \quad(5,6) \\
2(11,1) \\
2(11,1)\end{array}$ & $\begin{array}{l}0,189 \\
\mathbf{0 , 0 4 8} \\
0,094\end{array}$ \\
\hline $\begin{array}{l}\text { Otras complicaciones } \\
\text { Insuficiencia cardiaca } \\
\text { Insuficiencia renal } \\
\text { Evisceración } \\
\text { Abdomen abierto } \\
\text { Otras }\end{array}$ & $\begin{array}{rr}5 & (1,2) \\
7 & (1,7) \\
4 & (0,9) \\
4 & (0,9) \\
18 & (4,3)\end{array}$ & $\begin{array}{lr}1 & (5,6) \\
5 & (5,6) \\
1 & (5,6) \\
1 & (5,6) \\
2 & (11,1)\end{array}$ & $\begin{array}{l}0,222 \\
0,285 \\
0,189 \\
0,189 \\
0,194\end{array}$ \\
\hline
\end{tabular}

Tabla 4. Análisis multivariante de factores de riesgo para COVID-19

\begin{tabular}{|llll|}
\hline & Odds ratio & IC 95\% & p valor \\
\hline Estancia posoperatoria & 1,035 & $1,007-1,065$ & $\mathbf{0 , 0 1 5}$ \\
Reintervención & 5,025 & $1,650-15,311$ & $\mathbf{0 , 0 0 5}$ \\
\hline
\end{tabular}


Cai et al., con un $26 \%$ de pacientes infectados tras cirugía emergente en Wuhan, China ${ }^{18}$. En Brescia, en el epicentro de la pandemia en Italia, Doglietto et al. ${ }^{19}$, informaron de una tasa de infección del $12,3 \%$ en pacientes sometidos a diversos procedimientos quirúrgicos. Otros estudios han mostrado incidencias inferiores en distintos subgrupos de pacientes: $6,34 \%$ en pacientes de cirugía torácica de Wuhan, China $^{20} ; 5,3 \%$ en pacientes con neoplasias ginecológicas y tratamiento oncológico activo en Milán, Italia $^{21}$ o $0,93 \%$ en cirugía pediátrica de pacientes de Estados Unidos $^{22}$. Nuestro $4,1 \%$ es similar al 7,35\% indicado por el Hospital Universitario La Paz (Madrid, España) ${ }^{23}$ y al 7\% del Hospital de la Princesa

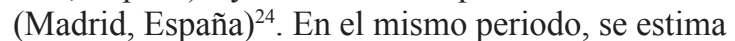
que SARS-CoV-2 infectó al 11,5\% de la población general de la Comunidad de Madrid ${ }^{25}$.

Sin embargo, COVID-19 es una importante amenaza para el paciente quirúrgico, asociando un $20 \%$ de mortalidad de forma consistente en numerosos estudios $^{7,12,19}$. Los primeros resultados durante la pandemia revelaron un exceso de mortalidad en cirugías habituales, como colecistectomía o reparación de hernia inguinal ${ }^{8}$. En el estudio de Lei et al. ${ }^{7}$, los pacientes intervenidos con infección posoperatoria por COVID-19 requerían en un 44,1\% de los casos ingreso en UCI, por SDRA, shock o infección secundaria, y su tasa de mortalidad era del 20,6\%. Este exceso de mortalidad se debe fundamentalmente a las complicaciones respiratorias en el periodo posoperatorio. De acuerdo con los resultados del estudio CovidSurg ${ }^{12}$, las complicaciones pulmonares sucedieron en un $51,2 \%$ de los pacientes: $40,4 \%$ presentaron neumonía, $21,3 \%$ requirieron ventilación y un $14,4 \%$ tuvieron SDRA. La mortalidad global fue del $23,8 \%$, siendo las complicaciones pulmonares responsables de un exceso de mortalidad del 38\%. Finalmente, en el estudio de Brescia de Doglietto et al. ${ }^{19}$, el 43,9\% de los pacientes COVID-19 padecieron neumonía y el 14,63\% SDRA, ambos porcentajes superiores que en grupo sin COVID-19
$(2,44 \%$ y $1,22 \%$, respectivamente $)$ y la tasa de mortalidad posoperatoria fue del 19,51\%.

La estancia hospitalaria prolongada y la necesidad de reintervención son factores de riesgo para la infección por COVID-19. Muchos de los pacientes intervenidos a finales de febrero e inicios de marzo vieron que su periodo posoperatorio transcurrió en el peor momento de la historia. Puesto que ningún país puede descartar una segunda o tercera ola en los próximos meses, debemos mantener todas las medidas de precaución aprendidas en este periodo y minimizar las complicaciones posoperatorias, permitiendo así un periodo posoperatorio seguro para nuestros pacientes.

\section{Conclusión}

Las intervenciones durante la pandemia de COVID-19 resultaron en una incidencia baja de COVID-19, aunque con una elevada mortalidad posoperatoria. La decisión de intervenir debe ser valorada frente al riesgo adicional para el paciente en contextos de elevada transmisión comunitaria.

\section{Responsabilidades éticas}

Protección de personas y animales. Los autores declaran que para esta investigación no se han realizado experimentos en seres humanos ni en animales.

Confidencialidad de los datos. Los autores declaran que en este artículo no aparecen datos de pacientes.

\section{Conflictos de interés: no hay}

Este estudio fue aprobado por el Comité de Ética de Investigación (número de registro: 134-20) y realizado de acuerdo con los estándares éticos de la Declaración de Helsinki de 1975.

\section{Bibliografía}

1. World Health Organization. WHO announces COVID-19 outbreak a pandemic. [Actualizado el 12 de marzo de 2020; acceso el 10 de enero de 2021]. Disponible en: http://www.euro.who. int/en/health-topics/healthemergencies/ coronavirus-covid-19/news/news/2020/3/ whoannounces-covid-19-outbreak-apandemic.
2. World Health Organization, Coronavirus disease (COVID-2019). Weekly epidemiological update. [Actualizado el 12 de enero de 2021; acceso el 19 de enero de 2021] Disponible en: http://www.who.int/emergencies/ diseases/novel-coronavirus-2019/ situation-reports.

3. Johns Hopkins Coronavirus Resource Center - COVID-19 Map. [Actualizado el 19 de enero de 2021; acceso el 19 de enero de 2021]. Disponible en: https:// coronavirus.jhu.edu/map.html.

4. Ministerio de Sanidad, Gobierno de España. Enfermedad por el coronavirus (COVID-19) - Actualización nº 292. [Actualizado el 18 de enero de 2021; acceso el 19 de enero de 2021]. https:// www.mscbs.gob.es/profesionales/ saludPublica/ccayes/alertasActual/ nCov/documentos/Actualizacion_292 COVID-19.pdf. 
5. Sun P, Qie S, Liu Z, Ren J, Li K, Xi J. Clinical Characteristics of Hospitalized Patients With SARS-CoV-2 Infection: A Single Arm Meta-Analysis. J Med Virol. 2020 Feb 28;10.1002/jmv.25735. doi: $10.1002 / j m v .25735$.

6. Guan WJ, Liang WH, Zhao Y, Liang HR, Chen ZS, Li YM, et al. Comorbidity and its impact on 1590 patients with COVID-19 in China: a nationwide analysis. Eur Respir J 2020;55:2000547. doi: 10.1183/13993003.00547-2020.

7. Lei S, Jiang F, Su W, Chen C, Chen J, Mei W, et al. Clinical Characteristics and Outcomes of Patients Undergoing Surgeries During the Incubation Period of COVID-19 Infection. EClinicalMedicine 2020;5;21:100331. doi: 10.1016/j. eclinm.2020.100331.

8. Aminian A, Safari S, Razeghian-Jahromi A, Ghorbani M, Delaney CP. COVID-19 Outbreak and Surgical Practice: Unexpected Fatality in Perioperative Period. Ann Surg 2020 Mar 26. doi: 10.1097/SLA.0000000000003925.

9. Bartlett DL, Howe JR, Chang G, Crago A, Hogg M, Karakousis G, et al. Management of Cancer Surgery Cases During the COVID-19 Pandemic: Considerations. Ann Surg Oncol 2020;27:1717-20. doi: 10.1245/s10434020-08461-2.

10. Balibrea JM, Badia JM, Rubio Pérez I, Martín Antona E, Álvarez Peña E, García Botella S, et al. Surgical Management of Patients With COVID-19 Infection. Recommendations of the Spanish Association of Surgeons. Cir Esp. 2020;98:251-9. doi: 10.1016/j. ciresp.2020.03.001.

11. COVIDSurg Collaborative. Elective Surgery Cancellations Due to the COVID-19 Pandemic: Global Predictive Modelling to Inform Surgical Recovery Plans. Br J Surg. 2020 May 12. doi: 10.1002/bjs.11746.

12. COVIDSurg Collaborative. Mortality and pulmonary complications in patients undergoing surgery with perioperative SARS-CoV-2 infection: an international cohort study. Lancet. 2020;4;396(10243):27-38. doi: 10.1016/ S0140-6736(20)31182-X.

13. von Elm E, Altman DG, Egger M, Pocock SJ, Gøtzsche PC, Vandenbroucke JP. STROBE Initiative. The Strengthening the Reporting of Observational Studies in Epidemiology (STROBE) statement: guidelines for reporting observational studies. J Clin Epidemiol. 2008;61:344-9. doi: 10.1016/j.jclinepi.2007.11.008.

14. Dindo D, Demartines N, Clavien PA. Classification of surgical complications: a new proposal with evaluation in a cohort of 6336 patients and results of a survey. Ann Surg. 2004;240:205-13. doi: 10.1097/01.sla.0000133083.54934.ae.

15. Tilmans G, Chenevas-Paule Q, Muller X, Breton A, Mohkam K, Ducerf C, et al. Surgical Outcomes After Systematic Preoperative SARS-CoV-2 Screening. Surgery. 2020 May 18. doi: 10.1016/j. surg.2020.05.006

16. Khan J, van Boxe Gl, Mercer S. Is Minimal Access Surgery Possible and Safe During the COVID-19 Pandemic? Br J Surg. 2020;107:e268. doi: 10.1002/ bjs.11731.

17. Lombardi CP, D'Amore A, Grani G, Ramundo V, Boscherini M, Gordini L, et al. Endocrine Surgery During COVID-19 Pandemic: Do We Need an Update of Indications in Italy? Endocrine. 2020;4:1-4. doi: 10.1007/s12020-02002357-7.

18. Cai M, Wang G, Zhang L, Gao J, Xia Z, Zhang P, et al. Performing Abdominal Surgery During the COVID-19 Epidemic in Wuhan, China: A Single-Centred, Retrospective, Observational Study. Br J Surg. 2020;107:e183-e185. doi: 10.1002/ bjs.11643.

19. Doglietto F, Vezzoli M, Gheza F, Lussardi GL, Domenicucci M, Vecchiarelli L, et al. Factors Associated With Surgical Mortality and Complications Among
Patients With and Without Coronavirus Disease 2019 (COVID-19) in Italy. JAMA Surg. 2020 Jun 12. doi: 10.1001/ jamasurg.2020.2713.

20. Li YK, Peng S, Li LQ, Wang Q, Ping W, Zhang N, et al. Clinical and Transmission Characteristics of Covid-19 - A Retrospective Study of 25 Cases From a Single Thoracic Surgery Department. Curr Med Sci. 2020;40:295-300. doi: 10.1007/ s11596-020-2176-2.

21. Bogani G, Ditto A, Bosio S, Brusadelli C, Raspagliesi F. Cancer patients affected by COVID-19: Experience from Milan, Lombardy. Gynecol Oncol. 2020;158:2625. doi: 10.1016/j.ygyno.2020.06.161.

22. Lin EE, Blumberg TJ, Adler AC, Fazal FZ, Talwar D, Ellingsen K, et al. Incidence of COVID-19 in Pediatric Surgical Patients Among 3 US Children's Hospitals. JAMA Surg. 2020;155:775-7. doi: 10.1001/jamasurg.2020.2588.

23. Álvarez Gallego M, Gortázar de Las Casas S, Migueláñez P, Rubio-Pérez I, Barragán Serrano C, Álvarez Peña E, et al. SARS-CoV-2 Pandemic on the Activity and Professionals of a General Surgery and Digestive Surgery Service in a Tertiary Hospital. Cir Esp. 2020;98:320-7. doi: 10.1016/j.ciresp.2020.04.001.

24. Di Martino M, García Septiem J, Maqueda González R, Muñoz de Nova JL, de la Hoz Rodríguez A, Correa Bonito A, et al. Elective Surgery During the SARS-CoV-2 Pandemic (COVID-19): A Morbimortality Analysis and Recommendations on Patient Prioritisation and Security Measures. Cir Esp. 2020 Apr 29;S0009739X(20)30166-4. doi: 10.1016/j. ciresp.2020.04.029.

25. Pollán M, Pérez-Gómez B, PastorBarriuso R, Oteo J, Hernán MA, Pérez-Olmeda M, et al. Prevalence of SARS-CoV-2 in Spain (ENE-COVID): a nationwide, population-based seroepidemiological study. Lancet 2020;396(10250):535-44. doi: 10.1016/ S0140-6736(20)31483-5. 